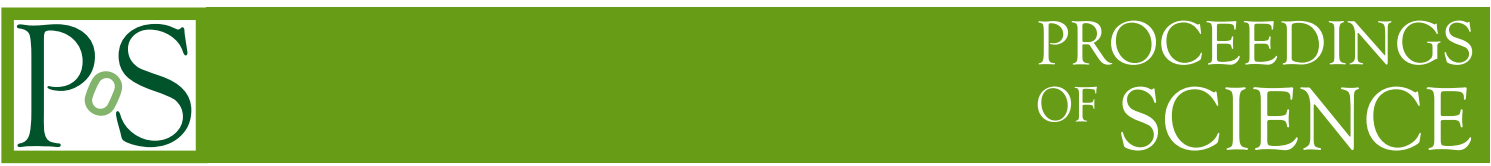

\title{
Probing QCD with top-quark pairs at CMS
}

\author{
Katerina LIPKA* on behalf of the CMS Collaboration \\ Deutsches Elektronen-Synchrotron \\ Notkestrasse 85, D-22607, Hamburg, Germany \\ E-mail: katerina.lipka@desy.de
}

The inclusive cross section for top-pair production as measured by the CMS experiment in protonproton collisions at a center-of-mass of $7 \mathrm{TeV}$ at the LHC is compared to the QCD prediction at highest order available, using various sets of parton distribution functions. For each parton distribution set, the pole mass of the top quark, and the strong coupling constant, are extracted. This extraction provides an important test of the mass scheme applied in Monte Carlo simulations and gives complementary information, with different sensitivity to theoretical and experimental uncertainties, than direct measurements of top-quark mass. The determined strong coupling is in a good agreement with the world average. This is the first determination of the strong coupling using top-quark production.

The European Physical Society Conference on High Energy Physics

18-24 July, 2013

Stockholm, Sweden

${ }^{*}$ Speaker. 
Top-quark pairs at the LHC are produced predominantly through gluon-gluon fusion. The cross section for top-pair production, $\sigma_{t \bar{t}}$, depends on the value of the top mass, $m_{t}$, the strong coupling, $\alpha_{S}\left(M_{Z}\right)$, and the gluon distribution, $g(x)$. Here, the recent results [1] on the determination of $m_{t}^{\text {pole }}$ and $\alpha_{S}\left(M_{Z}\right)$ using the CMS measurement [2] of the top-pair production cross section at $\sqrt{7} \mathrm{TeV}$ are presented. The measurement is used together with the QCD prediction [3] of $\sigma_{t \bar{t}}$ at next-to-next-to-leading order (NNLO) with soft-gluon resummation performed at next-to-nextleading-log (NNLL). In Fig. 1 the dependence of $\sigma_{t \bar{t}}$ on $m_{t}^{\text {pole }}$ and $\alpha_{S}\left(M_{Z}\right)$ is presented. Different PDF sets are used, each of those provided for various values of $\alpha_{S}\left(M_{Z}\right)$. Both $m_{t}$ and $\alpha_{S}\left(M_{Z}\right)$ alter the $\sigma_{t \bar{t}}$ prediction such that any variation of one parameter can be compensated by a variation of the other. Therefore simultaneous extraction of $\alpha_{S}\left(M_{Z}\right)$ and $m_{t}$ is not possible. For the determination of $m_{t}^{\text {pole }}$, the strong coupling is fixed to the world average, $\alpha_{S}\left(M_{Z}\right)=0.1184 \pm 0.0007$ [4]. For the determination of $\alpha_{S}\left(M_{Z}\right), m_{t}$ is fixed to the Tevatron average, $m_{t}=173.18 \pm 0.94 \mathrm{GeV}$ [5] with an additional uncertainty of $1 \mathrm{GeV}[6]$ due to the assumption $m_{t}^{M C} \equiv m_{t}^{\text {pole }}$.
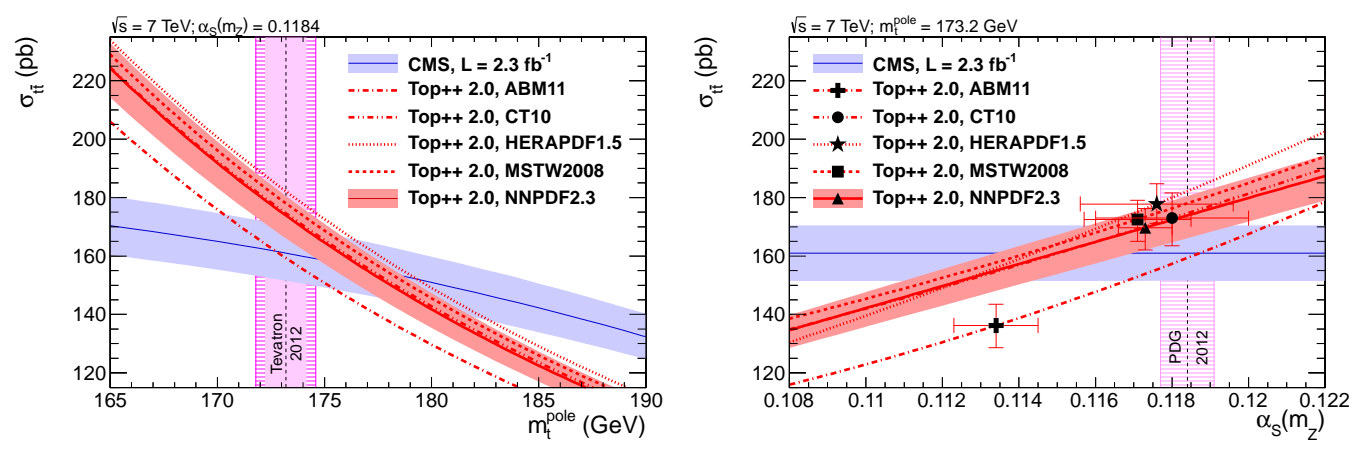

Figure 1: Predicted top-pair cross section at NNLO+NNLL, as a function of the top-quark mass (left) and of the strong coupling constant (right), using five different NNLO PDF sets, compared to the cross section measured by CMS. The uncertainties on the measured $\sigma_{t \bar{t}}$ and on the prediction with NNPDF2.3 are illustrated with filled bands, while the uncertainties on the $\sigma_{t \bar{t}}$ predictions using the other PDF sets are indicated only at the corresponding default $\alpha_{S}\left(M_{Z}\right)$ values. The $m_{t}$ and $\alpha_{S}\left(M_{Z}\right)$ regions favored by the direct measurements at the Tevatron and by the latest world average, respectively, are shown as hatched areas.

The values of $m_{t}^{\text {pole }}$ and $\alpha_{S}\left(M_{Z}\right)$ are obtained from the maximum of the marginalized posterior:

$$
P(x)=\int f_{\exp }\left(\sigma_{t \bar{t}} \mid x\right) f_{\mathrm{th}}\left(\sigma_{t \bar{t}} \mid x\right) d \sigma_{t \bar{t}}, \quad x=m_{t}^{\text {pole }}, \alpha_{S}\left(M_{Z}\right)
$$

The Gaussian probability, $f_{\exp }\left(\sigma_{t \bar{t}}\right)$, represents the measured cross section and its uncertainty. The probability function $f_{\mathrm{th}}\left(\sigma_{t \bar{t}}\right)$ for the predicted $\sigma_{t \bar{t}}$ is shown in Fig. 2 (left) and is obtained from a convolution of two probability distributions: the Gaussian distribution accounting for the PDF uncertainty; and the rectangular function that provides equal probability over the whole range covered by the scale variation $0.5 \mu_{0}<\mu_{r}, \mu_{f}<2 \mu_{0}$ with $\mu_{0}=m_{t}^{\text {pole }}$. An example of $P\left(\alpha_{S}\right)$ is shown in Fig. 2. The confidence interval is determined from the $68 \%$ area around the maximum of the posterior and requiring equal function values at the left and right edges.

In Fig. 3, the values of the top-quark pole mass, $m_{t}^{\text {pole }}$, determined using fixed $\alpha_{S}\left(M_{Z}\right)$, and of $\alpha_{S}\left(M_{Z}\right)$ obtained when fixing the value of $m_{t}^{\text {pole }}$, using five different NNLO PDF sets are shown. Using NNPDF2.3, $m_{t}^{\text {pole }}=176.7_{-3.4}^{+3.8} \mathrm{GeV}$ and the strong coupling $\alpha_{S}\left(M_{Z}\right)=0.1151_{-0.0032}^{+0.0033}$ are 

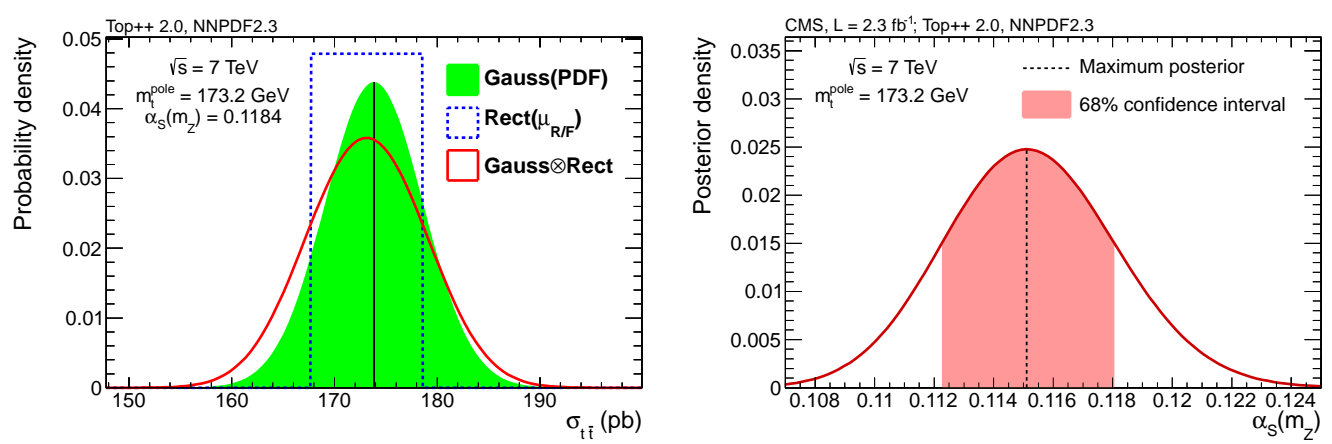

Figure 2: Left: probability distributions for the predicted $\sigma_{t \bar{t}}$ at NNLO+NNLL and the NNPDF2.3 parton distributions. The resulting probability (solid line) is obtained by convolving a Gaussian distribution (filled area) that accounts for the PDF uncertainty with a rectangular function (dashed line) that covers the scale variation uncertainty. Right: Marginal posterior $P\left(\alpha_{S}\right)$ obtained with the NNPDF2.3 parton distributions.

obtained. This is the first determination of the strong coupling constant from top-quark production and the first $\alpha_{S}\left(M_{Z}\right)$ result at full NNLO QCD obtained at a hadron collider.
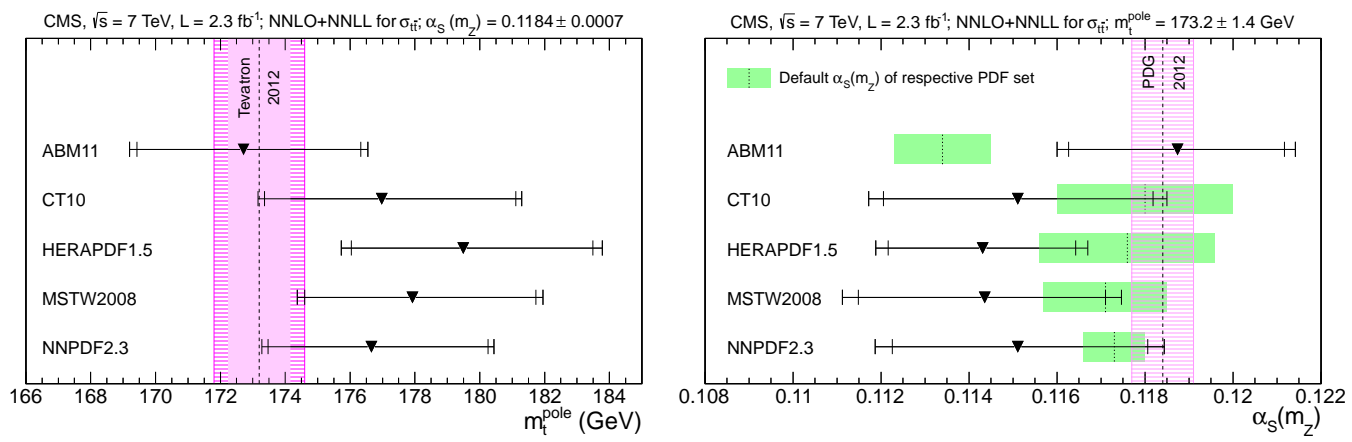

Figure 3: The resulting $m_{t}^{\text {pole }}$ (left) and $\alpha_{S}\left(M_{Z}\right)$ (right) using $\sigma_{t \bar{t}}$ with different PDF sets The inner error bars include the uncertainties on the measured cross section and on the LHC beam energy as well as the PDF and scale uncertainties on the predicted cross section. The outer error bars additionally account for the uncertainty on the $\alpha_{S}\left(M_{Z}\right)$ or $m_{t}$ world average. For comparison, the latest average of direct $m_{t}$ measurements at the Tevatron and the world average $\alpha_{S}\left(M_{Z}\right)$ are shown with its uncertainty as hatched band. For each PDF set, the default $\alpha_{S}\left(M_{Z}\right)$ value and its uncertainty are indicated using a dotted line and a shaded band.

\section{References}

[1] [CMS Collaboration], CERN-PH-EP-2013-121 [arXiv:1307.1907].

[2] [CMS Collaboration], JHEP11 (2012) 067.

[3] M. Czakon, P. Fiedler, A. Mitov [arXiv:1303.6254], M. Czakon, A. Mitov, TOP++ [arXiv:1112.5675].

[4] [Particle Data Group], Phys. Rev. D 86 (2012) 010001.

[5] [CDF and D0 Collaborations], Phys. Rev. D 86 (2012) 092003.

[6] A. Buckley et al., Phys. Rept. 504 (2011) 145. 